\title{
Development of a Monocyte Activation Test as an Alternative to the Rabbit Pyrogen Test for Mono- and Multi-Component Shigella GMMA-Based Vaccines
}

\author{
Danielle Carson ${ }^{1, *(\mathbb{D}}$, Sophie Myhill ${ }^{1}$, Elena Palmieri ${ }^{2}{ }^{\oplus}$, Francesca Necchi ${ }^{2}$, Sjoerd Rijpkema ${ }^{1}\left({ }^{1}\right.$, \\ Francesca Micoli ${ }^{2}$, Ida Karin Nordgren ${ }^{1}$, Omar Rossi ${ }^{2, *},+\left[\right.$ and Caroline Vipond ${ }^{1, *,+}$ \\ 1 The National Institute for Biological Standards and Control (NIBSC), South Mimms EN6 3QG, UK; \\ sophie.myhill@nibsc.org (S.M.); Sjoerd.Rijpkema@nibsc.org (S.R.); Karin.Nordgren@nibsc.org (I.K.N.) \\ 2 GSK Vaccines Institute for Global Health (GVGH) S.r.l., via Fiorentina 1, 53100 Siena, Italy; \\ elena.x.palmieri@gsk.com (E.P.); francesca.x.necchi@gsk.com (F.N.); francesca.x.micoli@gsk.com (F.M.) \\ * Correspondence: danielle.carson@nibsc.org (D.C.); omar.x.rossi@gsk.com (O.R.); \\ caroline.vipond@nibsc.org (C.V.) \\ $+\quad$ O.R. and C.V. should be considered joint senior authors.
}

Citation: Carson, D.; Myhill, S.; Palmieri, E.; Necchi, F.; Rijpkema, S.; Micoli, F.; Nordgren, I.K.; Rossi, O.;

Vipond, C. Development of a

Monocyte Activation Test as an Alternative to the Rabbit Pyrogen Test for Mono- and Multi-Component Shigella GMMA-Based Vaccines. Microorganisms 2021, 9, 1375. https://doi.org/10.3390/ microorganisms 9071375

Academic Editor: Mickael Blaise

Received: 21 May 2021

Accepted: 15 June 2021

Published: 24 June 2021

Publisher's Note: MDPI stays neutra with regard to jurisdictional claims in published maps and institutional affiliations.

Copyright: (C) 2021 by the authors. Licensee MDPI, Basel, Switzerland. This article is an open access article distributed under the terms and conditions of the Creative Commons Attribution (CC BY) license (https:// creativecommons.org/licenses/by/ $4.0 /)$

\begin{abstract}
Generalised modules for membrane antigens (GMMA)-based vaccines comprise the outer membrane from genetically modified Gram-negative bacteria containing membrane proteins, phospholipids and lipopolysaccharides. Some lipoproteins and lipopolysaccharides are pyrogens; thus, GMMA-based vaccines are intrinsically pyrogenic. It is important to control the pyrogenic content of biological medicines, including vaccines, to prevent adverse reactions such as febrile responses. The rabbit pyrogen test (RPT) and bacterial endotoxin test (BET) are the most commonly employed safety assays used to detect pyrogens. However, both tests are tailored for detecting pyrogenic contaminants and have considerable limitations when measuring the pyrogen content of inherently pyrogenic products. We report the adaptation of the monocyte activation test (MAT) as an alternative to the RPT for monitoring the pyrogenicity of Shigella GMMA-based vaccines. The European Pharmacopoeia endorses three MAT methods (A-C). Of these, method $\mathrm{C}$, the reference lot comparison test, was identified as the most suitable. This method was evaluated with different reference materials to ensure parallelism and consistency for a mono- and multi-component Shigella GMMA vaccine. We demonstrate the drug substance as a promising reference material for safety testing of the matched drug product. Our results support the implementation of MAT as an alternative to the RPT and use of the defined parameters can be extended to GMMA-based vaccines currently in development, aiding vaccine batch release.
\end{abstract}

Keywords: outer membrane vesicle (OMV); generalised modules for membrane antigens (GMMA); monocyte activation test (MAT); reactogenicity; pyrogenicity

\section{Introduction}

Shigellae spp. are the causative agents of shigellosis, characterised by stomach cramps, fever and diarrhoea, which can sometimes be bloody [1]. According to a study on the Global Burden of Diseases, Injuries and Risk Factors in 2016, shigellosis results in an estimated 212,438 deaths worldwide annually, 63,713 of which occur in children under five years of age [2]. Most Shigella-associated morbidity occurs in low- and middle-income countries (LMICs). An increasing prevalence of resistance to antibiotic treatments has led to Shigella being placed on the World Health Organisation (WHO) anti-microbial resistance priority list, highlighting a need for other options to control this disease [3]. There are currently no widely available licensed vaccines against Shigella, and as such, it has been prioritised by the WHO Product Development for Vaccine Advisory Committee (PDVAC) with the aim of focussing product development and abating policy and implementation gaps to accelerate vaccine availability [4]. 
In recent years, the GSK Vaccines Institute for Global Health (GVGH) has developed generalised modules for membrane antigens (GMMA)-based Shigella vaccines. GMMA particles are outer membrane vesicles (OMVs) produced by genetically modified Gramnegative bacteria [5-7]. An investigational S. sonnei GMMA vaccine (1790GAHB) was produced from attenuated $S$. sonnei strain 53G $\Delta$ virG $\Delta$ tolR $\Delta h t r B$, which carries a deletion for the inner and outer membrane linkage protein TolR allowing enhanced blebbing of OMVs [8]. Shed GMMA particles have been detoxified by deletion of the late acyltransferase gene $h t r B$ in the parent strain resulting in penta- rather than hexa-acylated lipopolysaccharide [5,9]. Subsequent clinical trials have shown 1790GAHB to be well tolerated and immunogenic in adults from both Europe and Kenya, where shigellosis is endemic [10-12]. This success has led to the development of a four-component vaccine containing GMMAs from S. sonnei and three clinically relevant $S$. flexneri strains.

By their nature, GMMA particles include several toll-like receptor agonists, most notably lipopolysaccharide, and thus, are inherently pyrogenic or fever-inducing [9]. In order to limit the risk of febrile reactions following administration, it is important to control the number of pyrogenic agents in parenteral medicine including vaccines. Three tests for assessing the pyrogenic content of medicinal products are described by the European Pharmacopoeia (Ph. Eur). Historically, the 'gold standard' has been the rabbit pyrogen test (RPT); other tests are: the bacterial endotoxins test (BET), also known as the Limulus amoebocyte lysate test (LAL), and the monocyte activation test (MAT) [13-15].

For the RPT, the product is administered intra-venously and the rabbit is monitored for increased body temperature, which provides a qualitative indication of pyrogenicity. The historic establishment of the RPT resulted in it being considered the "industry standard" and its use consumes around 400,000 rabbits worldwide annually [16]. Furthermore, the RPT was originally designed to detect contamination in products that should be pyrogen free by delivering parenterals intravenously to enhance pyrogenic sensitivity. However, vaccines are most commonly delivered via other administration routes, such as intramuscularly in the case of Shigella GMMA. Implementation of the RPT for the safety testing of the meningococcal OMV-based vaccine Bexsero gave a measure of batch consistency that is prone to false-positive results $[17,18]$. An adaptation to deliver a full human dose of vaccine intramuscularly to the rabbit (mRPT) was developed for testing 1790GAHB. Results demonstrated an acceptable average temperature rise of $0.5^{\circ} \mathrm{C}$ in the first four hours post-immunisation, which subsequently diminished [8,19]. However, notable drawbacks to $\mathrm{mRPT}$ remain, including the use of animals and associated high biological variability, as well as species-dependent differences in TLR specificity, which may affect the translatability of results obtained in rabbits [20]. In recent years, increased scientific scrutiny surrounding the translatability and ethical implications of the RPT have led to increased uptake of the in vitro BET as a replacement.

The BET relies on a clotting reaction between factor $C$ sourced from the Limulus polyphemus or Tachypleus tridentatus horseshoe crab in the presence of endotoxin. However, both species are known to be endangered. To overcome this ethical issue, the use of recombinant factor $\mathrm{C}$ ( $\mathrm{rFC}$, based on the gene sequence of the horseshoe crab) and a fluorimetric end-point detection method was accepted into the Ph. Eur in 2021 [21,22]. However, the BET and the rFC method are limited as a safety test due to their inability to detect non-endotoxin pyrogens. As both the RPT and BET were designed to detect pyrogenic contaminants in medicines, their use for inherently pyrogenic vaccines also requires extensive dilution of the product to an established non-pyrogenic dose.

The MAT was first introduced in the 1980s and included in the Ph. Eur. in 2010. This test quantifies in vitro a defined cytokine released by human monocytic cells following incubation with the test material $[15,23,24]$. Three MAT methods, A, B and C, are described: method A and B are quantitative and semi-quantitative tests, respectively, that utilise endotoxin as a comparative reference, whilst method $C$ uses an established lot as a reference [15]. The MAT has benefits over the RPT and BET in that it employs the use of human cells and is, therefore, more physiologically relevant. It is also able to detect 
both endotoxin and non-endotoxin pyrogens and it can be implemented quantitatively. Furthermore, the use of the MAT falls in line with the '3Rs' principle of Replacement, Reduction and Refinement [25]. In 2014, The National Institute for Biological Standards and Control (NIBSC) developed the MAT for batch release testing of Bexsero. However, successful implementation of the MAT as a replacement for the RPT requires optimisation and validation of the assay for the defined test product [15].

The Future Vaccine Manufacturing Research (FVMR) hub is a strategic partnership with researchers, industry and national centres, which works towards optimising vaccine manufacture and development to reduce the cost and increase accessibility of vaccines in LMICs. Through a FVMR hub-led collaboration between GVGH and NIBSC, we report the development of the MAT as a replacement for the RPT to be implemented in the consistency/safety testing of Shigella GMMA-based vaccines.

\section{Materials and Methods}

\subsection{Preparation of GMMA Drug Substance}

GMMA drug substances (DS) were produced at $30 \mathrm{~L}$ scale from 2 mutants of Shigella sonnei 53G (called 1790 and 2929) and from mutants of three Shigella flexneri serotypes as previously described [26,27]. Purified GMMA were stored in saline (S. flexneri GMMA) or Tris buffer (S. sonnei GMMA) at $-70 \pm 10{ }^{\circ} \mathrm{C}$. For accelerated stability studies, DS have been stored for 3 months at $-20^{\circ} \mathrm{C}, 4^{\circ} \mathrm{C}$ or $37-40^{\circ} \mathrm{C}$ until testing.

\subsection{Formulation of the GMMA Drug Product}

1790GAHB has been prepared as previously described [8]. Other GMMA have been formulated by adsorption in $\mathrm{NaCl} 154 \mathrm{mM} \mathrm{NaH} \mathrm{PO}_{4} 20 \mathrm{mM} \mathrm{pH} 6.5$ on Alhydrogel at a final concentration of $0.7 \mathrm{mg} / \mathrm{mL} \mathrm{Al}^{3+}$. Monovalent 1790GAHB formulations were prepared at $200 \mu \mathrm{g} / \mathrm{mL}$ total protein, corresponding to $12 \mu \mathrm{g} / \mathrm{mL}$ O-antigen concentration. Four-component drug product (DP) included S. sonnei 2929-GMMA and the three S. flexneri GMMA each at $30 \mu \mathrm{g} / \mathrm{mL}$ O-antigen. Four different S. sonnei 1790-GMMA fresh formulations were prepared: one 1790GAHB FF01, containing the same GMP GMMA DS lot (stored at $-70 \pm 10^{\circ} \mathrm{C}$ ) as used for 1790GAHB SH15-001 clinical lot (stored at $4{ }^{\circ} \mathrm{C}$ for $>6$ years), and three S. sonnei 1790-GMMA DP (DP batch A, B and C) using three different GMMA DS batches (all produced at $30 \mathrm{~L}$ scale, stored at $-70 \pm 10^{\circ} \mathrm{C}$ ). DP have been stored at $4 \pm 2{ }^{\circ} \mathrm{C}$ or at 25 and $37-40^{\circ} \mathrm{C}$ for up to three months in the accelerated stability studies performed (afterwards $4 \pm 2{ }^{\circ} \mathrm{C}$ until testing).

\subsection{Preparation of Peripheral Blood Mononuclear Cells}

Blood samples were supplied in leukoreduction system chambers or apheresis cones from the Oxford blood transfusion centre (NHS Blood and Transplant, Oxford, UK) on the day of donation, and peripheral blood mononuclear cells (PBMC) were isolated on receipt at NIBSC. Full consent was given by the donors.

PBMC isolation was carried out as previously described [28]. Briefly, the bottom and top tube of the cone were cut, and blood was allowed to drip into $2 \times 50 \mathrm{~mL}$ falcon tubes. Each $50 \mathrm{~mL}$ falcon tube was topped up to $45 \mathrm{~mL}$ with Heparin ( $5 \mathrm{IU} / \mathrm{mL}$ final concentration) in complete medium (RPMI supplemented with $100 \mathrm{U} / \mathrm{mL}$ penicillin, $100 \mu \mathrm{g} / \mathrm{mL}$ streptomycin, $10 \mathrm{mM}$ HEPES, $1 \times$ MEM non-essential amino acids (NEAA), $2 \mathrm{mM}$ L-glutamine and $2 \%(v / v)$ human AB serum (Biowest, Nuaille, France)). The $90 \mathrm{~mL}$ total volume of blood-heparin medium solution was layered on $3 \times 17 \mathrm{~mL}$ of Histopaque-1077 (Sigma Aldrich, Gillingham, UK) in $50 \mathrm{~mL}$ falcon tubes. PBMCs were isolated by density gradient centrifugation, washed three times with complete medium and resuspended in complete medium. Cells were quantified with Trypan blue, aliquoted in 50\% human AB serum, 50\% cryoprotectant (RPMI, L-glutamine, $2 \mathrm{mM}$ L-glutamine, DMSO (16.28\%)) to a density of $3 \times 10^{7}$ cells $/$ vial and stored in nitrogen vapour until use. 


\subsection{Monocyte Activation Test}

PBMC aliquots were thawed as previously described into chilled thawing media (RPMI supplemented with $1 \%(v / v)$ human AB serum (Biowest, Nuaille, France) [29]. The suspension was centrifuged $\left(240 \times g, 8 \mathrm{~min}, 10^{\circ} \mathrm{C}\right.$ with minimal breaking), supernatant was discarded and cell pellet was resuspended in $10 \mathrm{~mL}$ of complete cell culture media (RPMI supplemented with $100 \mathrm{U} / \mathrm{mL}$ penicillin, $100 \mu \mathrm{g} / \mathrm{mL}$ streptomycin, $10 \mathrm{mM}$ HEPES, $1 \times$ MEM NEAAs, $2 \mathrm{mM}$ L-glutamine and $2 \%(v / v)$ human AB serum (Biowest, Nuaille, France)). An aliquot of cell suspension was counted using Trypan blue to measure cell number and viability. The remining cell suspension was then diluted to $1 \times 10^{6}$ cells $/ \mathrm{mL}$ in complete cell culture medium and $125 \mu \mathrm{L}$ was added per well of 96-well round-bottomed polypropylene cell culture plates (Corning, Flintshire, UK). GMMA vaccine drug product (DP) and/or drug substance (DS) were gently mixed, diluted to an initial donor-dependent working concentration in complete cell culture medium and two-fold serially diluted in a pyrogen-free 96 deep-well plate (Greiner Bio-One, Gloucestershire, UK). Endotoxin dilutions were prepared from a $2000 \mathrm{IU} / \mathrm{mL}$ stock solution of endotoxin International Standard (IS, NIBSC 10/178), vortexed for $2 \mathrm{~min}$, diluted to an initial working concentration of $5 \mathrm{IU} / \mathrm{mL}$ and two-fold serially diluted as described for the GMMA vaccine. A $1 \mathrm{mg} / \mathrm{mL}$ stock solution of Pam3CysSerLys4 (Pam3CSK4) (Invivogen, Toulouse, France) was diluted to an initial working concentration of $250 \mathrm{ng} / \mathrm{mL}$ in complete cell culture medium followed by 3 -fold serial dilutions. Following preparation, $125 \mu \mathrm{L}$ of the test sample was added to cell culture plates in quadruplicate. To determine the interference of the alum adjuvant, Alhydrogel was diluted 10-fold and tested in isolation or spiked with endotoxin at a final concentration of $0.1 \mathrm{IU} / \mathrm{mL}$. Moreover, $125 \mu \mathrm{L}$ of Endotoxin IS (final concentration of $0.5 \mathrm{IU} / \mathrm{mL}$ ) and complete medium alone were also added to the cell culture plates in quadruplicate as a positive and negative control, respectively. Plates were incubated at $37^{\circ} \mathrm{C}, 5 \% \mathrm{CO}_{2}$ for $18-21 \mathrm{~h}$ in a humid atmosphere.

Following incubation, supernatants were removed from the cell culture plate and stored at $-20^{\circ} \mathrm{C}$ or analysed immediately for interleukin-6 (IL-6) content by sandwich enzyme linked immunosorbent assay (ELISA) as previously described [30]. Briefly, supernatants (diluted 1/10 in complete medium) were analysed alongside IL-6 IS (NIBSC, 89/548) dilutions from 62.5 to $4000 \mathrm{pg} / \mathrm{mL}$ using an in-house mouse monoclonal capture and sheep polyclonal (directly conjugated to horseradish peroxidase, HRP) detection antibody pair. Tetramethylbenzidine was used as the HRP substrate and the reaction stopped using $1 \mathrm{M} \mathrm{H}_{2} \mathrm{SO}_{4}$. The absorbance was measured at $450 \mathrm{~nm}$ (using $540 \mathrm{~nm}$ as a reference) on the Multiskan GO microplate reader (Thermo Fisher Scientific, Loughborough, UK).

\subsection{Data Analysis}

Outliers within the four replicates for each sample or reference dilution were excluded by Dixon's $Q$ test $(\alpha=0.02)$. CombiStats v. 6.0 (European Directorate for the Quality of Medicines \& HealthCare (EDQM), Council of Europe, Strasbourg, France) was used to determine endotoxin equivalents $(\mathrm{EE}, \mathrm{EU} / \mu \mathrm{g}$ protein) or relative pyrogenicity units (RPUs) and validity criteria. For RPU calculations, the reference standard was assigned an arbitrary value of 1 and a relative value was calculated for test samples. Sample results were considered valid if: regression; $p<0.05$, non-linearity; $p>0.001$, non-parallelism $p>0.001$ relative to the reference batch. EE and RPUs were determined from IL- 6 values for a minimum of six dilution points by four-parameter fit analysis.

\section{Results}

\subsection{MAT Method A and B Cannot Be Used to Test the Shigella GMMA Vaccine}

To investigate the suitability of Ph. Eur. MAT method A or B, which both utilise endotoxin as a comparative reference, S. sonnei GMMA DP and DS were analysed using cryopreserved PBMCs from four independent donors and compared to the endotoxin IS. Individual rather than pooled donors were tested in order to capture donor variability for this novel vaccine. Given that the GMMA DP is formulated with Alhydrogel, we first 
confirmed that the adjuvant did not interfere with the release of IL-6 by PBMCs or its detection. For this purpose, endotoxin was spiked (at a final concentration of $0.1 \mathrm{IU} / \mathrm{mL}$ ) into 10-fold serial dilutions of Alhydrogel. Results confirmed no effect of Alhydrogel on the release of IL-6 (Figure S1). The safety profile of the 1790GAHB SH15-001 clinical lot of S. sonnei GMMA DP has previously been established in adults [10-12]. As the 1790GAHB SH15-001 lot was $>6$ years old, S. sonnei GMMA DP was freshly formulated from the 1790-GMMA DS that was used to produce the clinical lot and has been stored at $-80^{\circ} \mathrm{C}$. This lot was termed 1790GAHB FF01 (FF01) and tested utilising endotoxin as a reference. A nine-point two-fold dose response curve was defined for S. sonnei GMMA DP (FF01) with a range of 0.003 and $12.5 \mathrm{ng} / \mathrm{mL}$ total protein (Figure 1). The starting concentration for DP and DS was optimised for each donor due to the variable response to Shigella GMMA. For the endotoxin IS reference, a nine-point two-fold dose response curve ranging from 0.02 to 5 endotoxin units (IU / mL) was used for all donors (Figure 1). Results showed significant non-parallelism for two out of four donors (Table 1). In addition, inter-donor variability for endotoxin equivalents was high, with a geometric coefficient of variation (GCV) of $227.77 \%$. Notably, inter-donor variability was independent of the endotoxin reference as it was also observed across the dose response curves for S. sonnei GMMA DP (FF01). Donor C163 was identified as a high IL-6 responder and required a further 6-fold dilution of the GMMA DP compared to the other three donors. Endotoxin stimulates IL-6 release via TLR4, whereas 1790GAHB GMMA have been shown to primarily activate TLR2 [9]. When TLR1/2 agonist, Pam3CysSerLys4 (Pam3CSK4), was added to the PBMCs of the four donors, IL-6 responses mirrored those observed with S. sonnei GMMA DP (FF01) (Figure S2a). However, analysis of $S$. sonnei GMMA DP and DS against Pam3CSK4 as a reference also revealed significant non-parallelism in two out of four donors, suggesting that the $S$. sonnei GMMA stimulates PBMCs via a combination of TLRs (Figure S2b, Table S1). Therefore, we concluded that methods A and B are unsuitable to analyse the reactivity of Shigella GMMA by MAT.
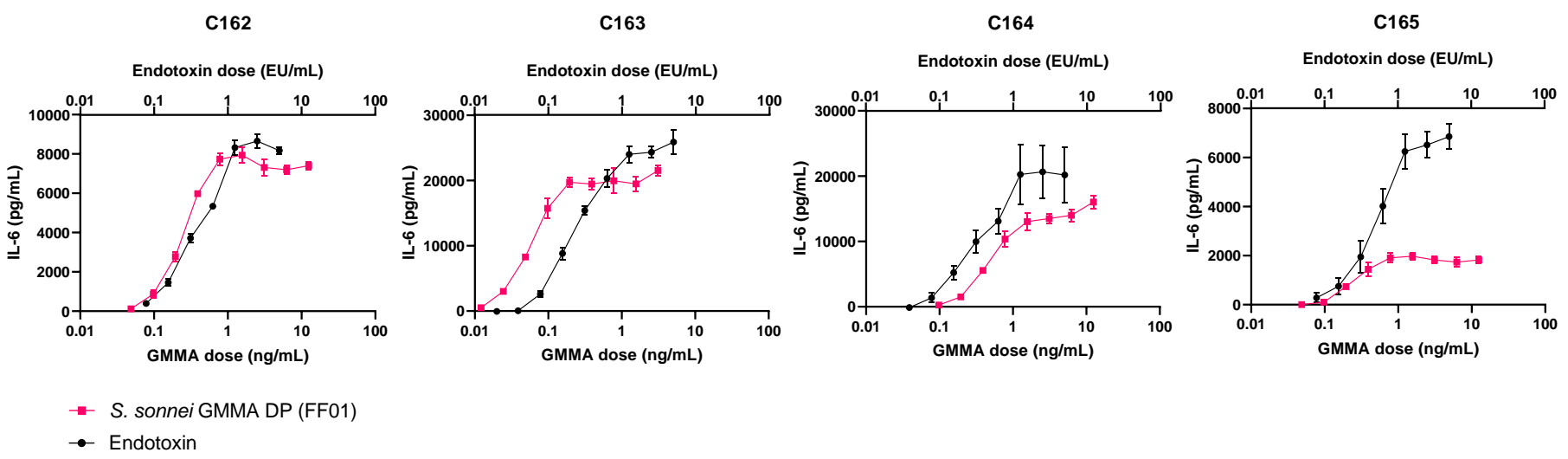

Figure 1. Dose response curves of peripheral blood mononuclear cells from four donors (C162-165) treated with $S$. sonnei GMMA drug product (DP, 1790GAHB FF01, pink squares) or endotoxin international standard (black circles). The highest concentration of S. sonnei GMMA DP was $12.5 \mathrm{ng} / \mathrm{mL}$ for donors C162, 164 and 165, and 3.125 ng/mL for donor C163.

Table 1. Endotoxin equivalents (EE, IU/ $/ \mathrm{g}$ protein) and statistical analysis for individual donors in the monocyte activation test.

\begin{tabular}{cccccc}
\hline Donor & EE (IU/ $\mu \mathrm{g})$ & NP $^{\mathbf{1}}(\boldsymbol{p}$-Value) & NL $^{\mathbf{2}}(\boldsymbol{p}$-Value) & Equivalence Ratio with Standard & Correlation $(r$, Weighted) \\
\hline C162 & 1419.4 & 0.000 & 0.000 & 1.291 & 0.949 \\
C163 & 3137.6 & 0.263 & 0.050 & 0.914 & 0.956 \\
C164 & 384.47 & 0.075 & 0.174 & 0.779 & 0.904 \\
C165 & 235.57 & 0.000 & 0.000 & -0.163 & 0.879 \\
\hline
\end{tabular}

${ }^{1} \mathrm{NP}$, non-parallelism; ${ }^{2} \mathrm{NL}$, non-linearity. 


\subsection{The GMMA Drug Product Demonstrates a Decrease in IL-6 Release over Time}

Further experiments focused on implementing Ph. Eur. method C: the reference lot comparison test. Relative pyrogenicity was calculated from a minimum of six of the nine dilutions modelled by four-parameter fit analysis using Combistats version 6.0 (EDQM, Council of Europe). Therefore, we aimed to implement 1790GAHB FF01 as a reference standard. S. sonnei GMMA DP 1790GAHB FF01 was compared to 1790GAHB DP SH15001 as well as three further $S$. sonnei 1790 GMMA DP batches (A, B and C), which were independently formulated from three separate batches of $S$. sonnei GMMA DS, to assess the batch-to-batch variability. DP batches A-C were all found to be within two-fold difference of S. sonnei GMMA DP (FF01) and had an inter-donor GCV of 32\%, $18 \%$ and $35 \%$ for batch A, B and C, respectively (Figure 2, Table 2 and Table S2).
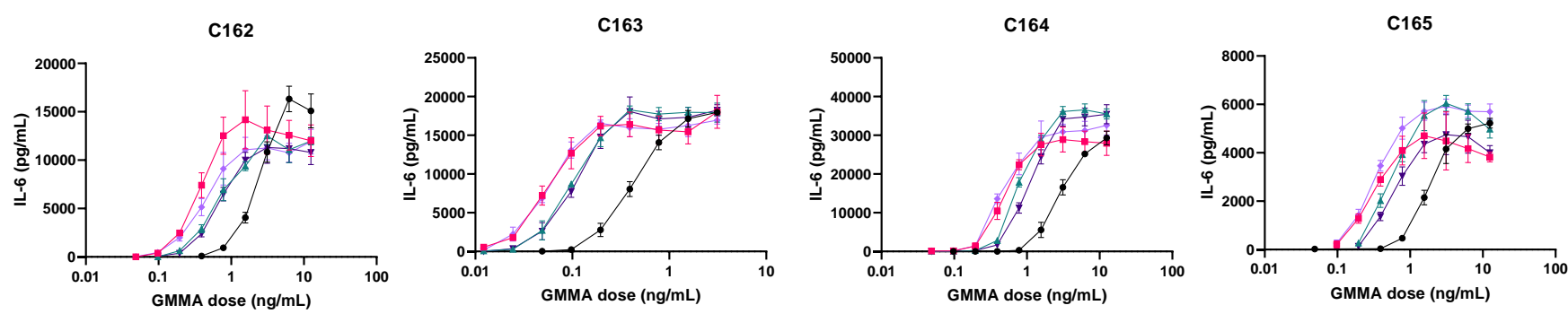

- $\quad$ S. sonnei GMMA DP (1790GAHB SH15-001)

$\rightarrow$ S. sonnei GMMA DP (1790GAHB FF01)

^ S. sonnei GMMA DP batch A

$\rightarrow$ S. sonnei GMMA DP batch $B$

$\rightarrow$ S. sonnei GMMA DP batch $\mathrm{C}$

Figure 2. Dose response curves of peripheral blood mononuclear cells from four donors (C162-165) treated with the clinically tested S. sonnei GMMA drug product (DP, 1790GAHB SH15-001, black circles), S. sonnei GMMA DP (1790GAHB FF01, pink squares) or three independent GMMA DP batches (A: Green triangles, B: Dark purple inverted triangles, C: Light purple diamonds). The highest concentration of S. sonnei GMMA DPs was $12.5 \mathrm{ng} / \mathrm{mL}$ for donors C162, 164 and 165, and $3.125 \mathrm{ng} / \mathrm{mL}$ for donor C163.

Table 2. Monocyte activation test data showing the relationship of different $S$. sonnei GMMA drug products (DPs) with a proposed reference batch, 1790GAHB FF01.

\begin{tabular}{cccc}
\hline GMMA Vaccine DP & Donor Average RPU ${ }^{\mathbf{1}}$ & Fold Change & Inter-Donor GCV (\%) \\
\hline 1790GAHB SH15-001 & 0.17 & 5.71 & 14.04 \\
Batch A & 0.65 & 1.54 & 31.94 \\
Batch B & 0.53 & 1.91 & 18.17 \\
Batch C & 1.02 & 0.98 & 35.07 \\
\hline
\end{tabular}

Importantly, the 1790GAHB SH15-001 clinical lot was found to be 5.7 times less pyrogenic than the proposed reference, S. sonnei GMMA DP (FF01) (Table 2). As the DP is formulated with Alhydrogel, it is stored at $4{ }^{\circ} \mathrm{C}$. At the time of testing, the 1790GAHB SH15-001 lot had, therefore, been stored at $4{ }^{\circ} \mathrm{C}$ for $>6$ years. Thus, the loss in IL-6 release is likely to be associated with changes to the DP over time. Indeed, testing of Shigella fourcomponent GMMA DP samples subjected to accelerated degradation following exposure at $25^{\circ} \mathrm{C}$ and $40^{\circ} \mathrm{C}$ for 3 months did demonstrate a temperature-associated loss in IL-6 release of 1.4-fold and 5.2-fold, respectively, compared to the sample stored at $4{ }^{\circ} \mathrm{C}$ (Figure 3a, Table S3). Furthermore, GMMA stored at $40^{\circ} \mathrm{C}$ showed a significant increase in the half maximal effective concentration (EC50) dose compared to GMMA stored at $4{ }^{\circ} \mathrm{C}$ (Figure $3 \mathrm{~b}$ ). The use of the DP as a reference batch would therefore require frequent monitoring and re-validation of fresh batches making a DP stored over a long period of time unsuitable as a reference standard. 
(a)

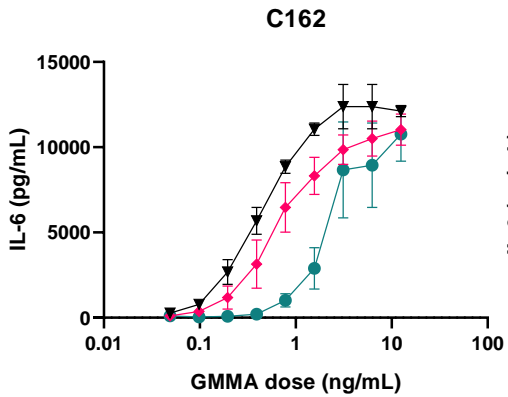

C164

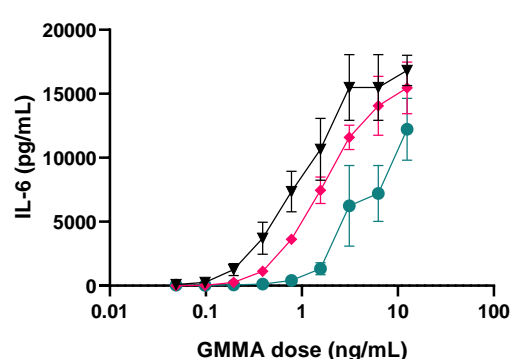

GMMA dose $(\mathrm{ng} / \mathrm{mL})$
C163

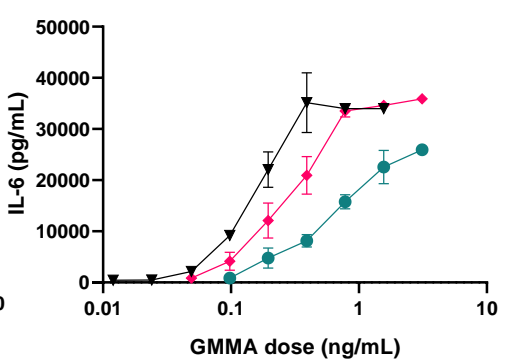

C165

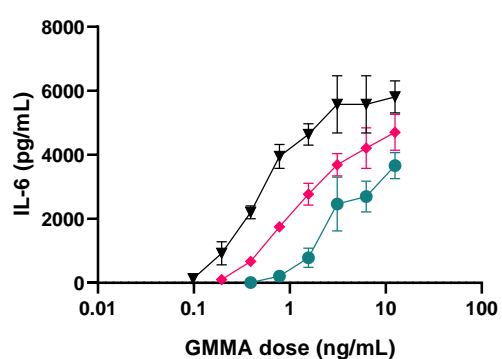

(b)

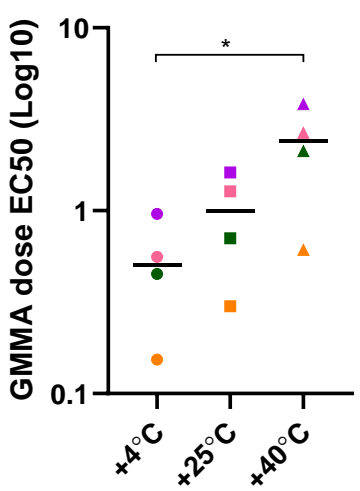

Shigella 4-component GMMA DP

- Shigella 4-component GMMA DP $4^{\circ} \mathrm{C}$

- Shigella 4-component GMMA DP $25^{\circ} \mathrm{C}$

-- Shigella 4-component GMMA DP $40^{\circ} \mathrm{C}$

Figure 3. (a) Dose response curves of peripheral blood mononuclear cells from four representative donors, C162-165 $(n=10)$, treated with Shigella four-component GMMA drug product (DP) stored at $4{ }^{\circ} \mathrm{C}$ (black inverted triangles), $25^{\circ} \mathrm{C}$ (pink diamonds) or $40^{\circ} \mathrm{C}$ for 3 months. The highest concentration of Shigella four-component GMMA DP was $12.5 \mathrm{ng} / \mathrm{mL}$ for donors C162, 164 and 165, and $3.125 \mathrm{ng} / \mathrm{mL}$ for donor C163. A two-way ANOVA $(p>0.05)$ was carried out on OD values for statistical analysis. (b) Half maximal effective dose (EC50) values from the interleukin-6 response of four donors (each donor is represented by a different colour). A one-way ANOVA test was carried out $\left({ }^{*} p<0.05\right)$.

\subsection{IL-6 Response to Shigella Drug Substance Is Comparable to Drug Product and Can Be} Proposed as a Reference for MAT Using Method C of Ph. Eur. Chapter 2.6.30

As prolonged storage in Alhydrogel at $4{ }^{\circ} \mathrm{C}$ affects the ability of the DP to induce IL-6 release from human PBMCs, we next investigated the DS as an alternative reference, which is not formulated with Alhydrogel and can be stored at $-80^{\circ} \mathrm{C}$. S. sonnei (1790GAHB FF01) and Shigella four-component GMMA DPs were tested using S. sonnei 1790 GMMA DS as a reference across PBMCs from 12 independent donors (Figure 4a and Table S4). On average, S. sonnei GMMA DP fell within a 0.61-fold range of the S. sonnei 1790 GMMA DS reference with an inter-donor GCV of 35.84\% and an average RPU of 1.63. Comparatively, the Shigella four-component GMMA DP fell within a 1.34-fold range of the S. sonnei 1790 GMMA DS reference with a higher inter-donor GCV of $47.93 \%$ and an average RPU of 0.75 . Of the 12 PBMC donors tested, parallelism between S. sonnei GMMA DP and the reference was observed in $100 \%$ of cases, whilst parallelism between the Shigella four-component GMMA DP and the reference was observed in only $25 \%$ of cases. These data indicate that S. sonnei 1790 GMMA DS is a suitable reference standard for S. sonnei GMMA vaccine DP only and is less suitable as a reference standard for multivalent Shigella GMMA vaccines. 
(a)
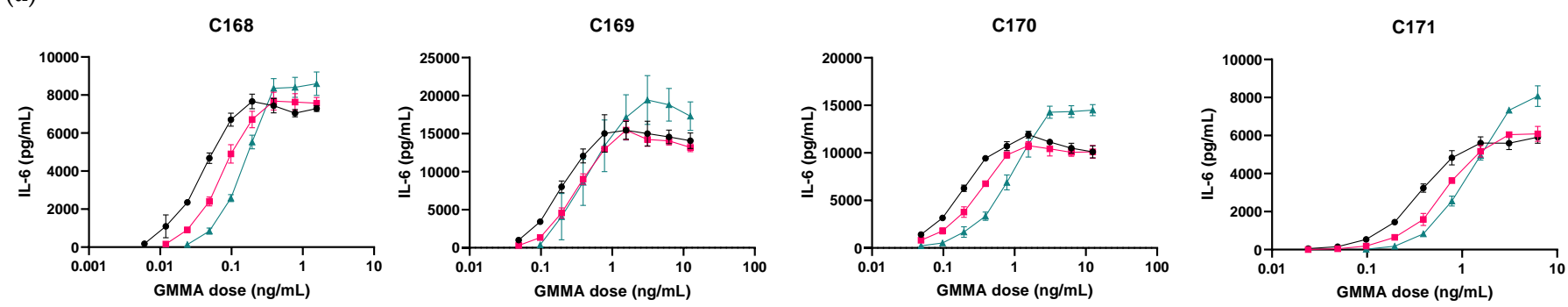

- S. sonnei 1790 GMMA DS

-- S. sonnei GMMA DP (1790GAHB FF01)

^- Shigella 4-component GMMA DP

(b)

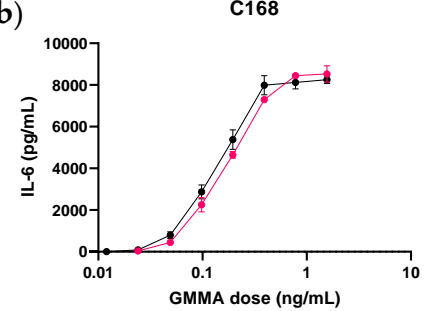

-- Shigella 4-component GMMA DS

- - Shigella 4-component GMMA DP
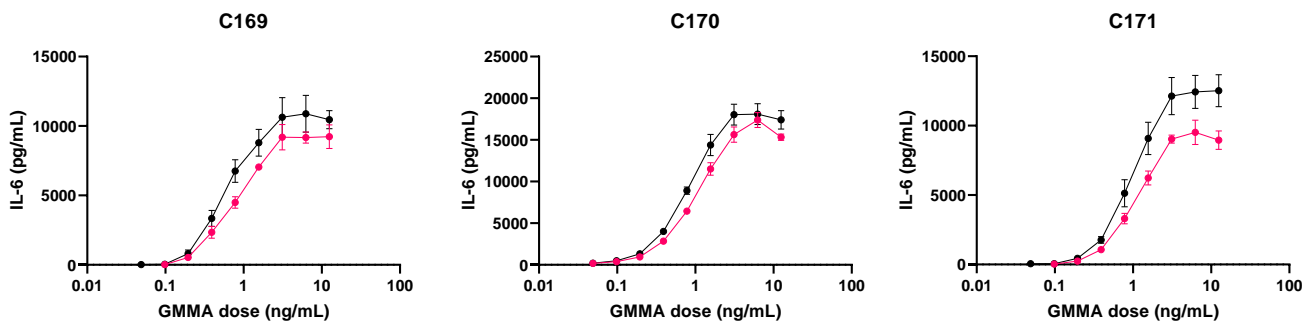

Figure 4. Dose response curves of peripheral blood mononuclear cells from four representative donors $(n>10)$; C 168-171 treated with S. sonnei GMMA (1790GAHB FF01) and Shigella four-component GMMA drug product (DP) and drug substance (DS). (a) Data show S. sonnei 1790 GMMA DS (pink sqaures), S. sonnei GMMA DP (1790GAHB FF01, black circles) and Shigella four-component GMMA DP (green triangles). The highest concentration of all Shigella GMMA vaccine products was $6.25 \mathrm{ng} / \mathrm{mL}$ for donors C169-171 and $1.56 \mathrm{ng} / \mathrm{mL}$ for donor C168. (b) Data show Shigella four-component DS (pink circles) and DP (black circles). The highest concentration of Shigella four-component GMMA DP and DS was $12.5 \mathrm{ng} / \mathrm{mL}$ for donors C169-171 and $1.56 \mathrm{ng} / \mathrm{mL}$ for donor C168.

We subsequently tested the Shigella four-component GMMA DS as a reference standard for Shigella four-component GMMA DP across PBMCs from 10 donors (Figure 4b, Table S5). On average, there was a 0.65 -fold difference between DS and DP across donors with an inter-donor GCV of $33.92 \%$ and an average RPU of 1.54. Parallelism between DS and DP was maintained across $100 \%$ of donors, supporting the implementation of the 'like-for-like' DS as the reference for its corresponding DP.

\subsection{Shigella GMMA Drug Substance Is More Stable Than the GMMA Drug Product}

To investigate the stability of the DS, Shigella GMMA DS samples were stored at their recommended storage temperature of $-80{ }^{\circ} \mathrm{C}$ or alternatively at $-20^{\circ} \mathrm{C}, 4^{\circ} \mathrm{C}$ and $37{ }^{\circ} \mathrm{C}$ for three months and tested on PBMCs from eight donors. Following incubation, no significant difference was observed in the dose response curves of samples stored at $-20{ }^{\circ} \mathrm{C}, 4{ }^{\circ} \mathrm{C}$ or $37^{\circ} \mathrm{C}$ compared to $-80^{\circ} \mathrm{C}$ in each individual donor (Figure 5a, Table S6). Furthermore, no significant difference was observed in the GMMA dose EC50 values (Figure 5b). A 1.1-, 1.5- and 1.6-fold decrease in IL-6 release was observed at $-20{ }^{\circ} \mathrm{C}, 4{ }^{\circ} \mathrm{C}$ and $37{ }^{\circ} \mathrm{C}$, respectively, compared to $-80^{\circ} \mathrm{C}$. These results demonstrate the increased stability of the Shigella GMMA DS over the DP. 
(a)

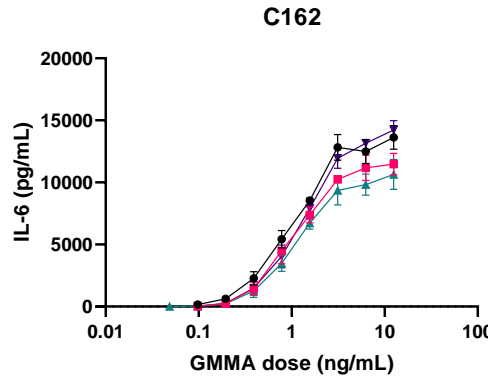

C164

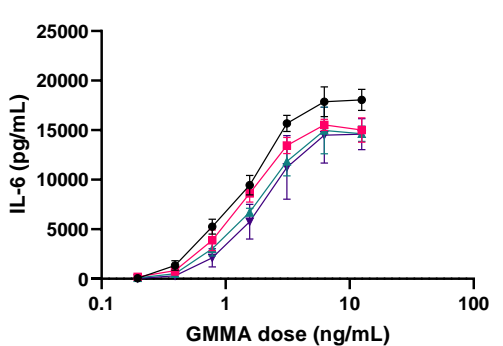

- Shigella 4-component GMMA DS $-80^{\circ} \mathrm{C}$

- Shigella 4-component GMMA DS $-20^{\circ} \mathrm{C}$

- Shigella 4-component GMMA DS $+4^{\circ} \mathrm{C}$

- Shigella 4-component GMMA DS $+37^{\circ} \mathrm{C}$
C163

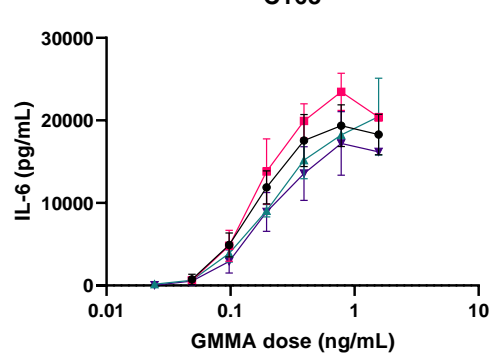

C165

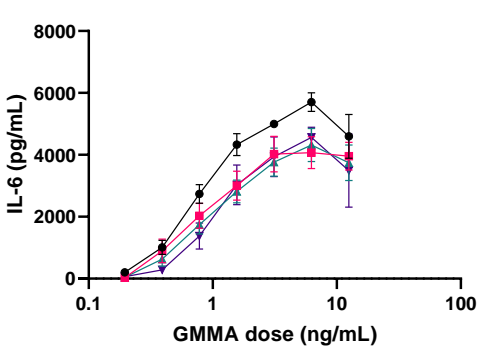

(b)

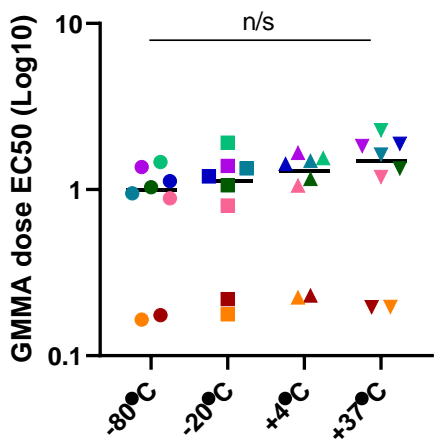

Shigella 4-component GMMA DS

Figure 5. (a) Dose response curves of peripheral blood mononuclear cells from four representative donors $(n=8)$ treated with Shigella four-component GMMA DS stored at $-80{ }^{\circ} \mathrm{C}$ (black circles), $-20{ }^{\circ} \mathrm{C}$ (pink squares), $4{ }^{\circ} \mathrm{C}$ (green triangles) and $37^{\circ} \mathrm{C}$ (purple inverted triangles). The highest concentration of Shigella four-component GMMA DP and DS was $12.5 \mathrm{ng} / \mathrm{mL}$ for donors C162, 164 and 165 and $1.56 \mathrm{ng} / \mathrm{mL}$ for donor C163. A two-way ANOVA $(p>0.05)$ was carried out on OD values for statistical analysis. (b) Half maximal effective dose (EC50) values from the interleukin-6 response of eight donors (each donor is represented by a different colour). A one-way ANOVA test was carried out $(p>0.05)$.

\section{Discussion}

The GMMA-based vaccine platform is an attractive approach to address a public health need in LMICs due to its simplicity in manufacture and strong immunogenic potential [31]. Promising phase I and II trials of the monovalent Shigella vaccine have led to the development of a four-component Shigella vaccine which will provide coverage against the most prevalent Shigella serotypes [10-12]. For vaccine batch release, it is important to confirm that the pyrogenic response is maintained at safe levels. Historic use of the RPT for batch release of Bexsero, an OMV-based meningococcal B vaccine, proved problematic due to inconsistencies and false-positive test results contributed by extensive dilution of the vaccine product due to its intrinsic pyrogenic properties [17,32]. In addition, BET is not a suitable replacement for OMV-based vaccines due to the complexity of pathogenassociated molecular patterns (PAMPs) and the inability of this test to detect pyrogens of non-endotoxin origin. Therefore, there is a requirement to develop a MAT for the batch release of GMMA-based vaccines.

It is important to develop and validate MATs according to specific requirements to ensure suitability of the method and several variables must be defined, including an appropriate reference standard, dilution range and cytokine for detection. A panel of cytokines released by human PBMCs after stimulation with $S$. sonnei GMMA was previously analysed and high levels of IL-6, tumour necrosis factor- $\alpha$, IL- $1 \beta$ and IL- 8 were shown to be expressed [9]. In 2014, NIBSC established the IL-6-MAT for batch release of Bexsero. This methodology utilises non-heat-inactivated human serum, which has been shown to be optimal over the use of heat-inactivated serum, which shows a loss of reactivity towards endotoxins [33]. Furthermore, IL-6 was selected as a readout due to its full secretion into the surrounding media and because its presence is a measure of both 
endotoxin and non-endotoxin contaminants [32,34]. Building on experience of the Bexsero MAT and knowledge of GMMA-stimulated cytokines, IL-6 was implemented here as a suitable readout for GMMA-based vaccines.

Method C: the reference lot comparison test was demonstrated as the most appropriate methodology for the Shigella GMMA vaccines following the observed non-parallelism between endotoxin and the vaccine, likely due to differences in the activated pattern recognition receptors (PRR). Indeed, whilst endotoxin is a known TLR4 agonist, previous studies have confirmed the induction of a primarily TLR2 response by detoxified Shigella GMMA [9]. Interestingly, non-parallelism was also observed with the TLR2/TLR1 ligand Pam3CSK4. Thus, GMMA-stimulated IL-6 release is likely contributed by a combination of PAMPs, highlighting the complexity of immunostimulatory components present on the surface of the GMMA. Furthermore, method C is more suitable for situations of increased donor variability as observed here.

In pyrogenicity studies in rabbits, it has been found that DPs are less reactogenic than DS. For this reason, 1790GAHB was investigated as a potential reference batch. Indeed, 1790GAHB DP has been confirmed to have a good safety profile based on clinical trials performed so far in adults. However, it was deemed unsuitable as a reference in the MAT due to the observed reduction in IL- 6 release over time, likely as a result of storage at $4{ }^{\circ} \mathrm{C}$ (due to formulation with alhydrogel). Studies have shown a reduction in rodent mortality when administered a mixture of endotoxin and aluminium hydroxide adjuvant compared to endotoxin alone $[35,36]$. It is possible that Alhydrogel masks GMMA by binding to exposed phosphate groups [36]. As Alhydrogel is only present in the DP, we used the 1790 GMMA DS as an alternative reference for S. sonnei GMMA DP. No interference of Alhydrogel with IL-6 release and detection was observed and similar RPUs were found for S. sonnei DS and DP. Whilst no significant difference was observed in the dose response curves of samples stored at increased temperatures compared to $-80^{\circ} \mathrm{C}$, a 1.6 -fold decrease in IL-6 release was observed for the DS stored at $37^{\circ} \mathrm{C}$, notably less than the 5.1-fold loss observed for the DP at $40{ }^{\circ} \mathrm{C}$. Further investigations are being carried out to establish the full stability profile of the DS in real time; however, the results demonstrate the improved stability of the DS over the DP and show its promise as the comparative reference.

As such, we propose the $S$. sonnei DS as a suitable reference for $S$. sonnei DP vaccine batches. The $S$. sonnei DS was not suitable as a reference for the Shigella four-component vaccine, likely due to the difference in PAMP composition and subsequent PRR signalling. However, our data demonstrate the parallelism, linearity and comparability of RPUs between Shigella four-component GMMA DS and DP. Therefore, our data support the implementation of Shigella four-component DS reference batch as a reference for Shigella four-component DP vaccine batches. We hope that forthcoming clinical trials with the Shigella four-component GMMA vaccine will demonstrate its safety, as previously observed for 1790GAHB. Future work aims to validate and implement this methodology to ensure the safety of vaccine batches utilised in planned clinical trials. The use of this methodology could be further extended to accelerate the clinical testing of other GMMA-based vaccines, including nontyphoidal Salmonella spp.

Supplementary Materials: The following are available online at https: / www.mdpi.com/article/ 10.3390/microorganisms9071375/s1, Figure S1: PBMC response to Alhydrogel; Figure S2: PBMC response to TLR1/2 ligand Pam3CSK4; Table S1: RPU and statistical analysis for GMMA with Pam3CKS4 as a reference standard; Table S2: RPU and statistical analysis for different batches of S. sonnei GMMA DP with a reference S. sonnei DP batch as standard; Table S3: RPU and statistical analysis of accelerated degradation Shigella 4-component GMMA drug product samples; Table S4: RPU and statistical analysis of mono- and multi-component Shigella GMMA drug products with S. sonnei GMMA drug substance as a reference standard; Table S5: RPU and statistical analysis of Shigella 4-component GMMA drug product with Shigella 4-component GMMA drug substance as a reference standard; Table S6: RPU and statistical analysis of accelerated degradation Shigella 4-component GMMA drug substance samples. 
Author Contributions: Conceptualisation, O.R., F.M., F.N., C.V. and I.K.N.; formal analysis, D.C. and S.M.; investigation, D.C. and S.M.; resources, E.P., F.N., F.M. and O.R.; writing-original draft preparation, D.C.; writing-review and editing, D.C., S.M., E.P., F.N., S.R., F.M, I.K.N., O.R. and C.V.; supervision, O.R., I.K.N. and C.V.; funding acquisition, F.M., S.R. and C.V. All authors have read and agreed to the published version of the manuscript.

Funding: This research is funded by the Department of Health and Social Care using UK Aid funding and is managed by the Engineering and Physical Sciences Research Council (EPSRC, grant number: $\mathrm{EP} / \mathrm{R} 013764 / 1)$. The views expressed in this publication are those of the author(s) and not necessarily those of the Department of Health and Social Care.

Institutional Review Board Statement: The study was conducted according to the guidelines of the Declaration of Helsinki, and approved by the Institutional Review Board (or Ethics Committee) of the National Institute of Biological Standards and Control (protocol code: 18/02/AN and date of approval: 14 Mar 2018).

Informed Consent Statement: Informed consent was obtained from all subjects involved in the study.

Data Availability Statement: Not applicable.

Acknowledgments: We thank Peter Rigsby for his advice with the statistical analysis.

Conflicts of Interest: GSK Vaccine Institute for Global Health Srl is an affiliate of GlaxoSmithKline Biologicals SA. E.P., O.R., F.N. and F.M. are employees of the GSK group of companies. The funders had no role in the design of the study; in the collection, analyses or interpretation of data; in the writing of the manuscript, or in the decision to publish the results.

Disclaimer: Bexsero is a trademark of the GSK group of companies.

\section{References}

1. Kotloff, K.L.; Riddle, M.S.; Platts-Mills, J.A.; Pavlinac, P.; Zaidi, A.K.M. Shigellosis. Lancet 2018, 391, 801-812. [CrossRef]

2. Khalil, I.; Troeger, C.; Blacker, B.F.; Rao, P.C.; Brown, A.; Atherly, D.; Brewer, T.G.; Engmann, C.M.; Houpt, E.R.; Kang, G.; et al. Morbidity and mortality due to shigella and enterotoxigenic Escherichia coli diarrhoea: The Global Burden of Disease Study 1990-2016. Lancet Infect. Dis. 2018, 18, 1229-1240. [CrossRef]

3. WHO. Global priority list of antibiotic-resistant bacteria to guide research, discovery, and development of new antibiotics. Fecha de Consult. 2017, 21, 7.

4. Giersing, B.K.; Vekemans, J.; Nava, S.; Kaslow, D.C.; Moorthy, V.; the WHO Product Development for Vaccines Advisory Committee. Report from the World Health Organization's third Product Development for Vaccines Advisory Committee (PDVAC) meeting, Geneva, 8-10th June 2016. Vaccine 2019, 37, 7315-7327. [CrossRef]

5. Maggiore, L.; Yu, L.; Omasits, U.; Rossi, O.; Dougan, G.; Thomson, N.R.; Saul, A.; Choudhary, J.; Gerke, C. Quantitative proteomic analysis of Shigella flexneri and Shigella sonnei Generalized Modules for Membrane Antigens (GMMA) reveals highly pure preparations. Int. J. Med Microbiol. 2016, 306, 99-108. [CrossRef] [PubMed]

6. Micoli, F.; Alfini, R.; Di Benedetto, R.; Necchi, F.; Schiavo, F.; Mancini, F.; Carducci, M.; Palmieri, E.; Balocchi, C.; Gasperini, G.; et al. GMMA Is a Versatile Platform to Design Effective Multivalent Combination Vaccines. Vaccines 2020, 8, 540. [CrossRef] [PubMed]

7. Scorza, F.B.; Colucci, A.M.; Maggiore, L.; Sanzone, S.; Rossi, O.; Ferlenghi, I.; Pesce, I.; Caboni, M.; Norais, N.; Di Cioccio, V.; et al. High Yield Production Process for Shigella Outer Membrane Particles. PLoS ONE 2012, 7, e35616.

8. Gerke, C.; Colucci, A.M.; Giannelli, C.; Sanzone, S.; Vitali, C.G.; Sollai, L.; Rossi, O.; Martin, L.B.; Auerbach, J.; Di Cioccio, V.; et al. Production of a Shigella sonnei Vaccine Based on Generalized Modules for Membrane Antigens (GMMA), 1790GAHB. PLoS ONE 2015, 10, e0134478. [CrossRef]

9. Rossi, O.; Pesce, I.; Giannelli, C.; Aprea, S.; Caboni, M.; Citiulo, F.; Valentini, S.; Ferlenghi, I.; MacLennan, C.A.; D’Oro, U.; et al. Modulation of endotoxicity of Shigella generalized modules for membrane antigens (GMMA) by genetic lipid A modifications: Relative activation of TLR4 and TLR2 pathways in different mutants. J. Biol. Chem. 2014, 289, 24922-24935. [CrossRef] [PubMed]

10. Obiero, C.W.; Ndiaye, A.G.W.; Sciré, A.S.; Kaunyangi, B.M.; Marchetti, E.; Gone, A.M.; Schütte, L.D.; Riccucci, D.; Auerbach, J.; Saul, A.; et al. A Phase 2a Randomized Study to Evaluate the Safety and Immunogenicity of the 1790GAHB Generalized Modules for Membrane Antigen Vaccine against Shigella sonnei Administered Intramuscularly to Adults from a Shigellosis-Endemic Country. Front. Immunol. 2017, 8, 1884. [CrossRef]

11. Launay, O.; Lewis, D.J.; Anemona, A.; Loulergue, P.; Leahy, J.; Sciré, A.S.; Maugard, A.; Marchetti, E.; Zancan, S.; Huo, Z.; et al. Safety Profile and Immunologic Responses of a Novel Vaccine Against Shigella sonnei Administered Intramuscularly, Intradermally and Intranasally: Results From Two Parallel Randomized Phase 1 Clinical Studies in Healthy Adult Volunteers in Europe. EBioMedicine 2017, 22, 164-172. [CrossRef] [PubMed] 
12. LLaunay, O.; Ndiaye, A.G.W.; Conti, V.; Loulergue, P.; Sciré, A.S.; Landre, A.M.; Ferruzzi, P.; Nedjaai, N.; Schütte, L.D.; Auerbach, J.; et al. Booster Vaccination With GVGH Shigella sonnei 1790GAHB GMMA Vaccine Compared to Single Vaccination in Unvaccinated Healthy European Adults: Results From a Phase 1 Clinical Trial. Front. Immunol. 2019, 10, 335. [CrossRef]

13. 10.0, E.P. Chapter 2.6.8 Rabbit Pyrogen Test; 2019; Volume 10. Available online: https://www.edqm.eu/en/europeanpharmacopoeia-ph-eur-10th-edition (accessed on 30 April 2021).

14. 10.0, E.P. Chapter 2.6.14 Bacterial Endotoxins; 2019; Volume 10. Available online: https://www.edqm.eu/en/europeanpharmacopoeia-ph-eur-10th-edition (accessed on 30 April 2021).

15. 10.0, E.P. Chapter 2.6.30 Monocyte-Activation Test; 2019; Volume 10. Available online: https://www.edqm.eu/en/europeanpharmacopoeia-ph-eur-10th-edition (accessed on 30 April 2021).

16. Hartung, T. The human whole blood pyrogen test-Lessons learned in twenty years. ALTEX 2015, 32, 79-100. [CrossRef]

17. Studholme, L.; Sutherland, J.; Desai, T.; Hockley, J.; Care, R.; Nordgren, I.K.; Vipond, C. Evaluation of the monocyte activation test for the safety testing of meningococcal B vaccine Bexsero: A collaborative study. Vaccine 2019, 37, 3761-3769. [CrossRef]

18. Valentini, S.; Santoro, G.; Baffetta, F.; Franceschi, S.; Paludi, M.; Brandini, E.; Gherardini, L.; Serruto, D.; Capecchi, B. Monocyteactivation test to reliably measure the pyrogenic content of a vaccine: An in vitro pyrogen test to overcome in vivo limitations. Vaccine 2019, 37, 3754-3760. [CrossRef]

19. Rossi, O.; Citiulo, F.; Mancini, F. Outer membrane vesicles: Moving within the intricate labyrinth of assays that can predict risks of reactogenicity in humans. Hum. Vaccines Immunother. 2021, 17, 601-613. [CrossRef]

20. Vaure, C.; Liu, Y. A Comparative Review of Toll-Like Receptor 4 Expression and Functionality in Different Animal Species. Front. Immunol. 2014, 5, 316. [CrossRef] [PubMed]

21. Piehler, M.; Roeder, R.; Blessing, S.; Reich, J. Comparison of LAL and rFC Assays-Participation in a Proficiency Test Program between 2014 and 2019. Microorganisms 2020, 8, 41. [CrossRef] [PubMed]

22. 10.3, E.P. Chapter 2.6.32 Test for Bacterial Endotoxins Using Recombinant Factor C; Supplement 10.3; 2020; Volume 10, p. 3. Available online: https:/ / www.edqm.eu/en/news/european-pharmacopoeia-supplement-103-now-available (accessed on 30 April 2021).

23. PPoole, S. Detection of pyrogen by cytokine release. Lancet 1988, 331, 130. [CrossRef]

24. Duff, G.W.; Atkins, E. The detection of endotoxin by in vitro production of endogenous pyrogen: Comparison with limulus amebocyte lysate gelation. J. Immunol. Methods 1982, 52, 323-331. [CrossRef]

25. Flecknell, P. Replacement, reduction and refinement. ALTEX 2002, 19, 73-78. [PubMed]

26. Palmieri, E.; Arato, V.; Oldrini, D.; Ricchetti, B.; Aruta, M.; Pansegrau, W.; Marchi, S.; Giusti, F.; Ferlenghi, I.; Rossi, O.; et al. Stability of Outer Membrane Vesicles-Based Vaccines, Identifying the Most Appropriate Methods to Detect Changes in Vaccine Potency. Vaccines 2021, 9, 229. [CrossRef]

27. Mancini, F.; Gasperini, G.; Rossi, O.; Aruta, M.G.; Raso, M.M.; Alfini, R.; Biagini, M.; Necchi, F.; Micoli, F. Dissecting the contribution of O-Antigen and proteins to the immunogenicity of Shigella sonnei generalized modules for membrane antigens (GMMA). Sci. Rep. 2021, 11, 1-10. [CrossRef]

28. Hoffmann, S.; Peterbauer, A.; Schindler, S.; Fennrich, S.; Poole, S.; Mistry, Y.; Montag-Lessing, T.; Spreitzer, I.; Löschner, B.; van Aalderen, M.; et al. International validation of novel pyrogen tests based on human monocytoid cells. J. Immunol. Methods 2005, 298, 161-173. [CrossRef]

29. Nordgren, I.K. Leukoreduction system chambers provide a valuable source of functional monocytes for the monocyte activation test by comparison with internationally validated methods. J. Immunol. Methods 2016, 428, 42-49. [CrossRef]

30. Findlay, L.; Eastwood, D.; Stebbings, R.; Sharp, G.; Mistry, Y.; Ball, C.; Hood, J.; Thorpe, R.; Poole, S. Improved in vitro methods to predict the in vivo toxicity in man of therapeutic monoclonal antibodies including TGN1412. J. Immunol. Methods 2010, 352, 1-12. [CrossRef] [PubMed]

31. Micoli, F.; MacLennan, C.A. Outer membrane vesicle vaccines. Semin. Immunol. 2020, 50, 101433. [CrossRef] [PubMed]

32. Vipond, C.; Sutherland, J.; Nordgren, K.; Kemp, G.; Heath, A.; Care, R.; Studholme, L. Development and validation of a monocyte activation test for the control/safety testing of an OMV-based meningococcal B vaccine. Vaccine 2019, 37, 3747-3753. [CrossRef]

33. Backer, M.W.A.M.-D. Performance of monocyte activation test supplemented with human serum compared to fetal bovine serum. ALTEX 2020. [CrossRef]

34. Solati, S.; Aarden, L.; Zeerleder, S.; Wouters, D. An improved monocyte activation test using cryopreserved pooled human mononuclear cells. Innate Immun. 2015, 21, 677-684. [CrossRef] [PubMed]

35. Norimatsu, M.; Ogikubo, Y.; Aoki, A.; Takahashi, T.; Watanabe, G.; Taya, K.; Sasamoto, S.; Tsuchiya, M.; Tamura, Y. Effects of aluminum adjuvant on systemic reactions of lipopolysaccharides in swine. Vaccine 1995, 13, 1325-1329. [CrossRef]

36. Shi, Y.; HogenEsch, H.; Regnier, F.E.; Hem, S.L. Detoxification of endotoxin by aluminum hydroxide adjuvant. Vaccine 2001, 19, 1747-1752. [CrossRef] 